Supporting Information

\title{
Gemini Peptide Amphiphiles with Broad-spectrum Antimicrobial Activity and Potent Anti-biofilm Capacity
}

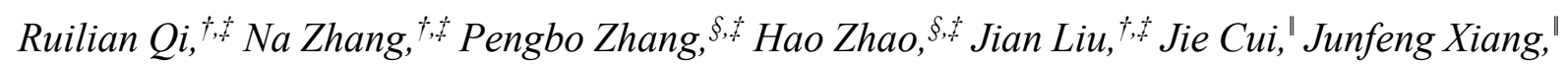
Yuchun Han, ${ }^{*}+$ Shu Wang, ${ }^{*}$, ,t. and Yilin Wang ${ }^{*},+,+$

${ }^{\dagger}$ Key Laboratory of Colloid, Interface and Chemical Thermodynamics, Beijing National Laboratory for Molecular Sciences (BNLMS), CAS Research/Education Center for Excellence in Molecular Sciences, Institute of Chemistry, Chinese Academy of Sciences, Beijing 100190, P. R. China

${ }^{\S}$ Key Laboratory of Organic Solids, Beijing National Laboratory for Molecular Sciences (BNLMS), CAS Research/Education Center for Excellence in Molecular Sciences, Institute of Chemistry, Chinese Academy of Sciences, Beijing 100190, P. R. China

"Center for Analysis and Testing, Institute of Chemistry, Chinese Academy of Sciences, Beijing 100190, P. R. China

†University of Chinese Academy of Sciences, Beijing 100049, P. R. China

\section{CORRESPONDING AUTHOR}

*hanyuchun@iccas.ac.cn; *wangshu@iccas.ac.cn; *yilinwang@iccas.ac.cn; 


\section{Table of contents}

1. Supplementary Figures $\quad$ S2

Figure S1 CFU number of $E$. coli after treatment with peptide amphiphiles $\quad$ S2

Figure S2 CFU number of $S$. aureus after treatment with peptide amphiphiles $\quad$ S3

Figure S3 CFU number of $C$. albicans after treatment with peptide amphiphiles $\quad$ S3

Figure S4 Cell viability of Hela cells after incubation with peptide amphiphiles $\quad$ S4

Figure S5 CLSM images of peptide amphiphiles-treated S. aureus biofilms $\quad$ S4

Figure S6 High-performance liquid chromatography results $\quad$ S5

$\begin{array}{ll}\text { Figure } \mathrm{S} 7{ }^{1} \mathrm{H} \text { NMR spectra } & \text { S6 }\end{array}$

$\begin{array}{ll}\text { Figure S8 ESI spectra } & \text { S7 }\end{array}$

2. ITC Analysis Process for Single Set of Identical Sites $\quad$ S8

\section{Supplementary Figures.}

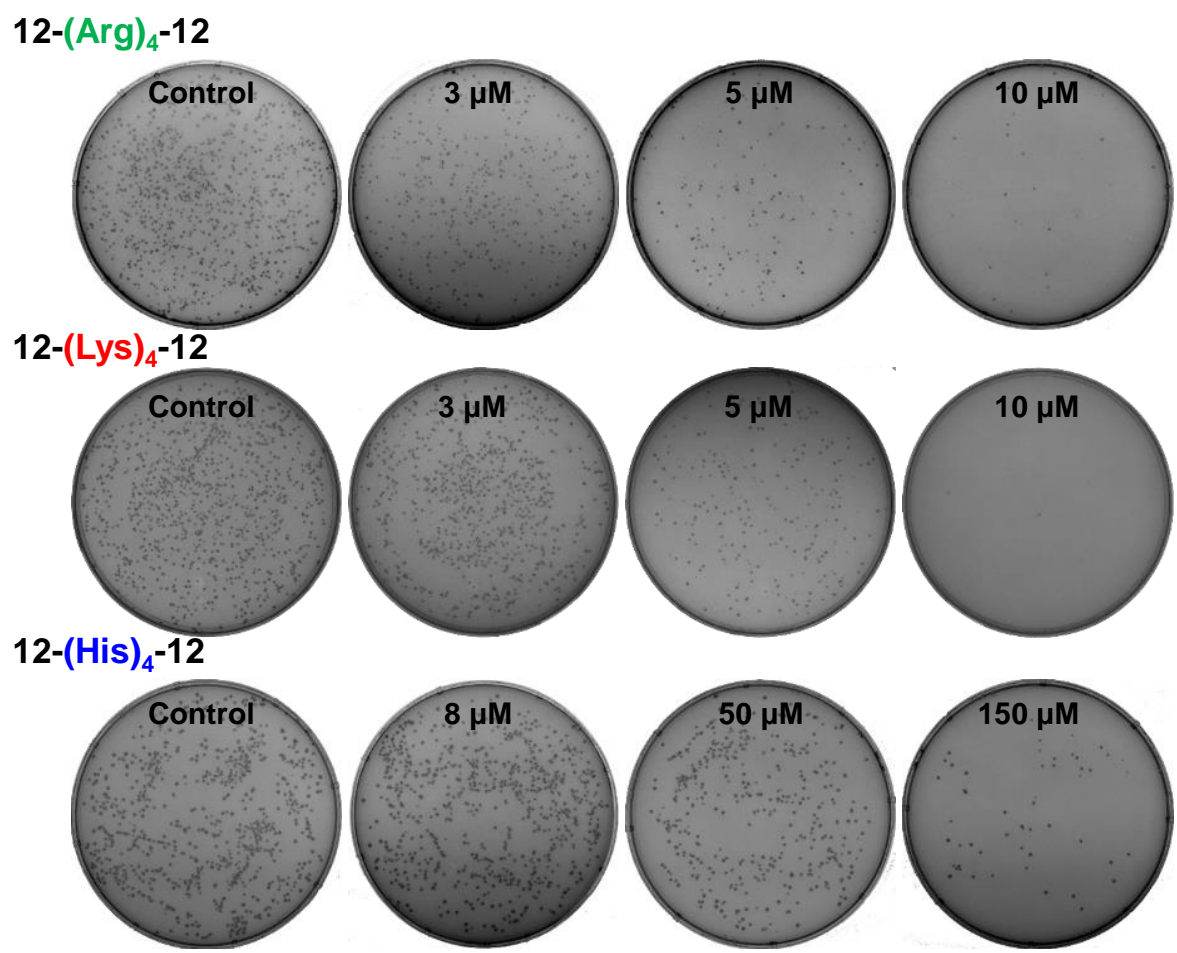

Figure S1. Number of colony forming units (CFU) of $E$. coli before (control) and after adding gemini peptide amphiphiles with different concentrations on LB agar plate 


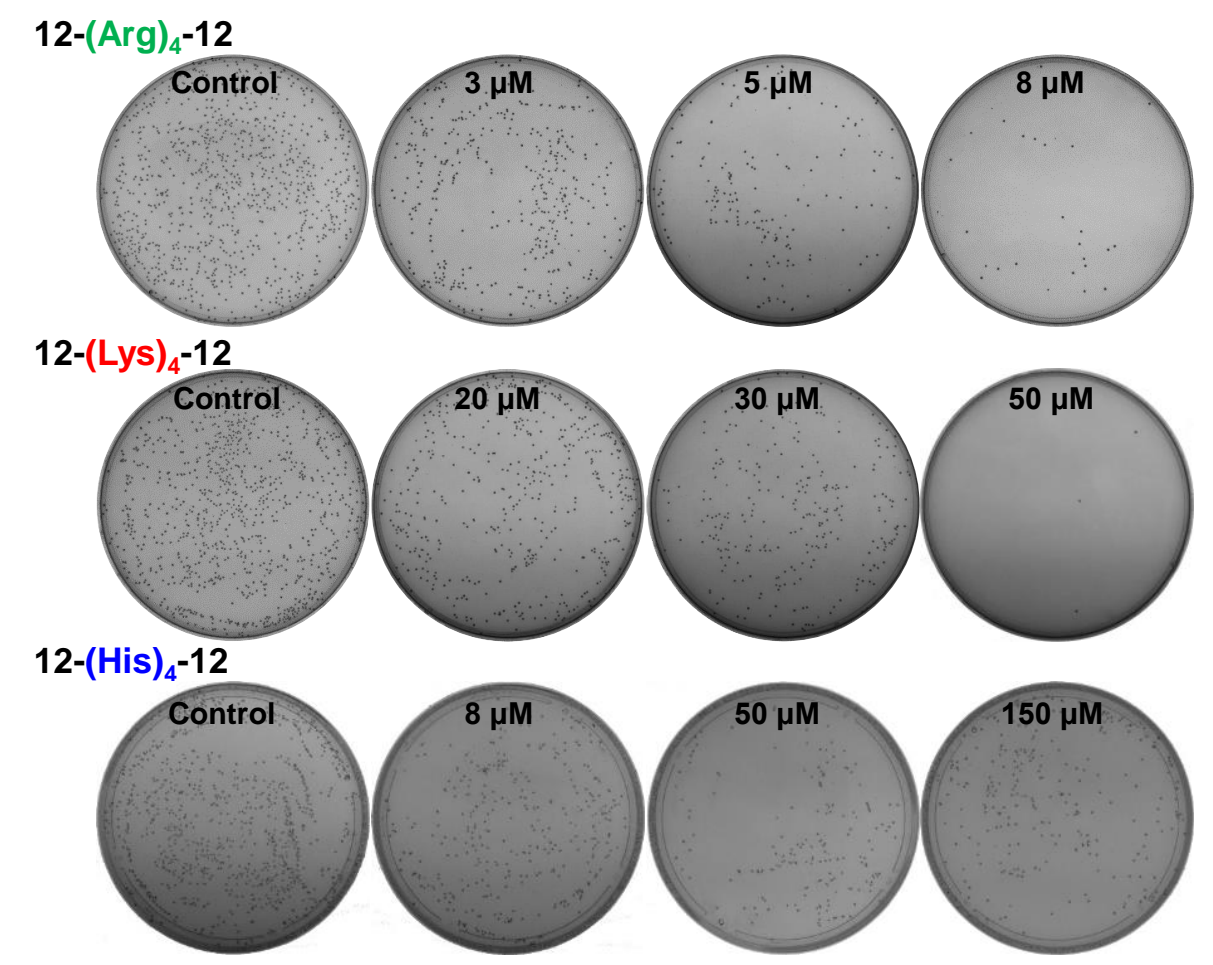

Figure S2. Number of colony forming units (CFU) of S. aureus before (control) and after adding gemini peptide amphiphiles with different concentrations on NB agar plate

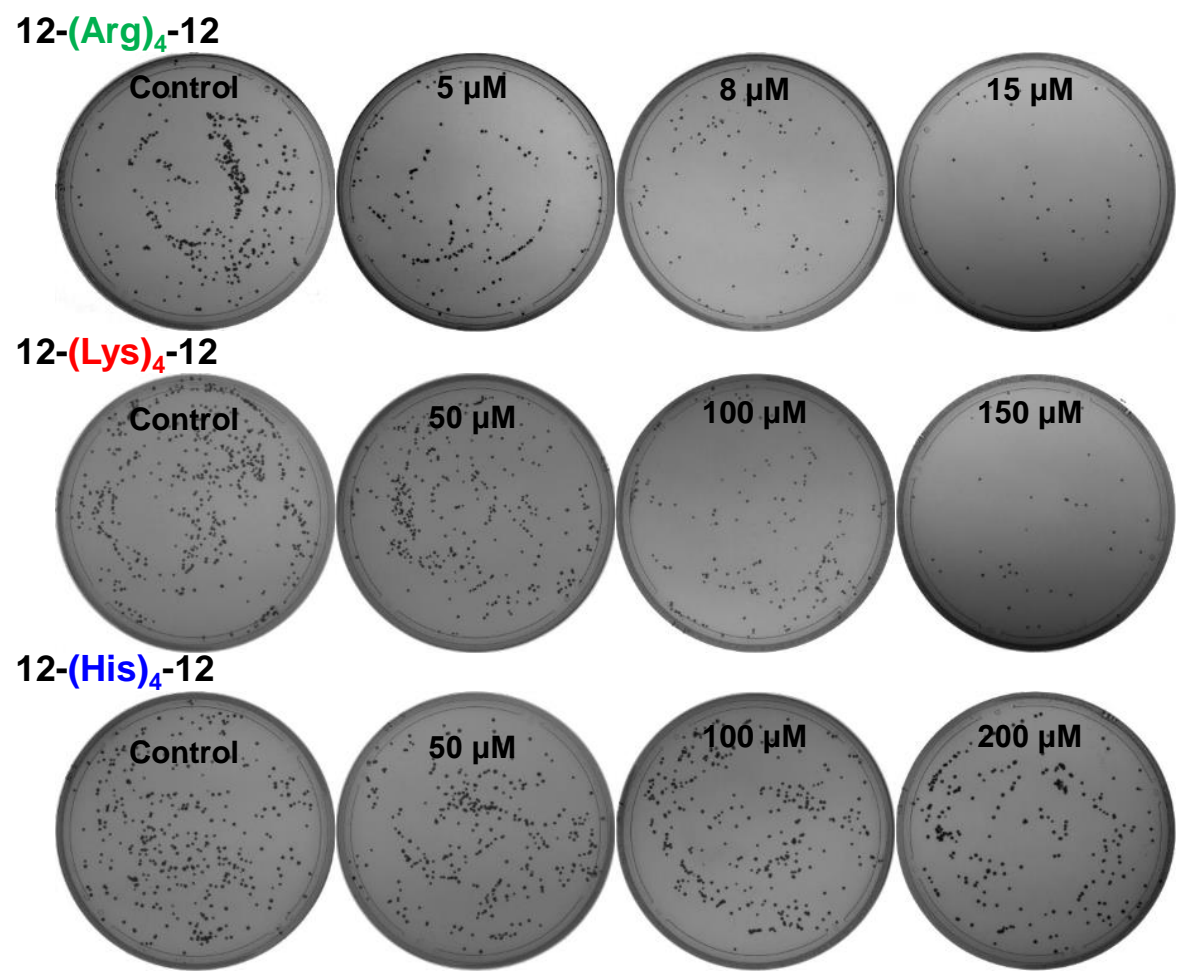

Figure S3. Number of colony forming units (CFU) of C. albicans before (control) and after adding gemini peptide amphiphiles with different concentrations on YPD agar plate. 


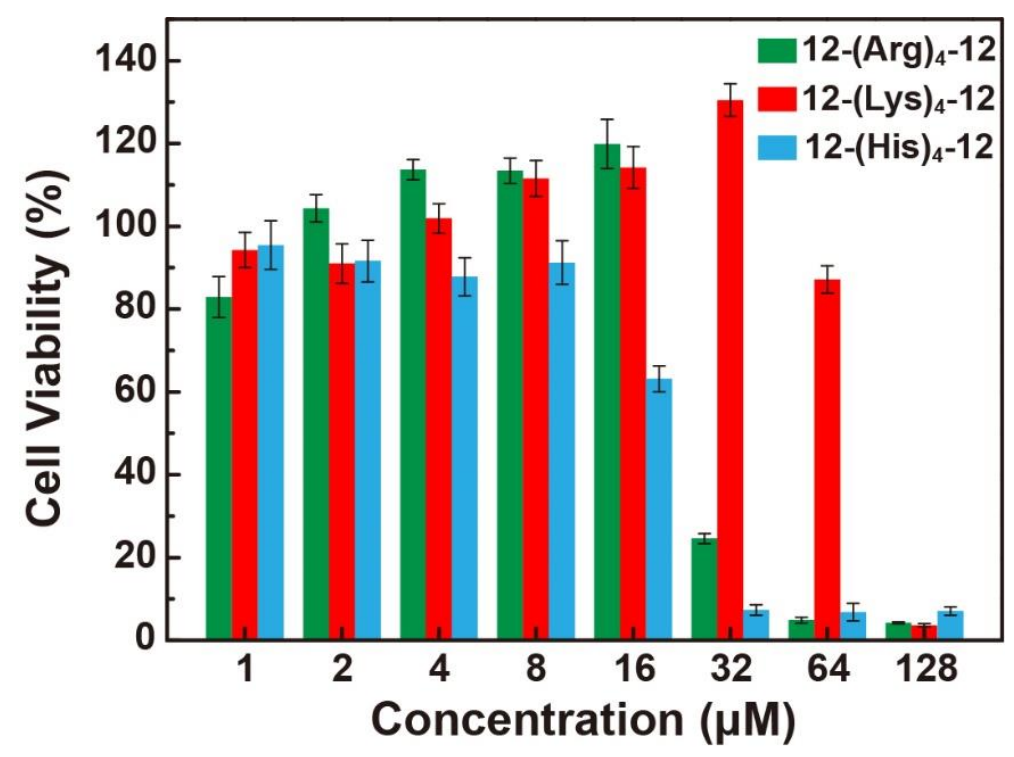

Figure S4. Cell viability of Hela cells after incubation with peptide amphiphiles at different concentrations.

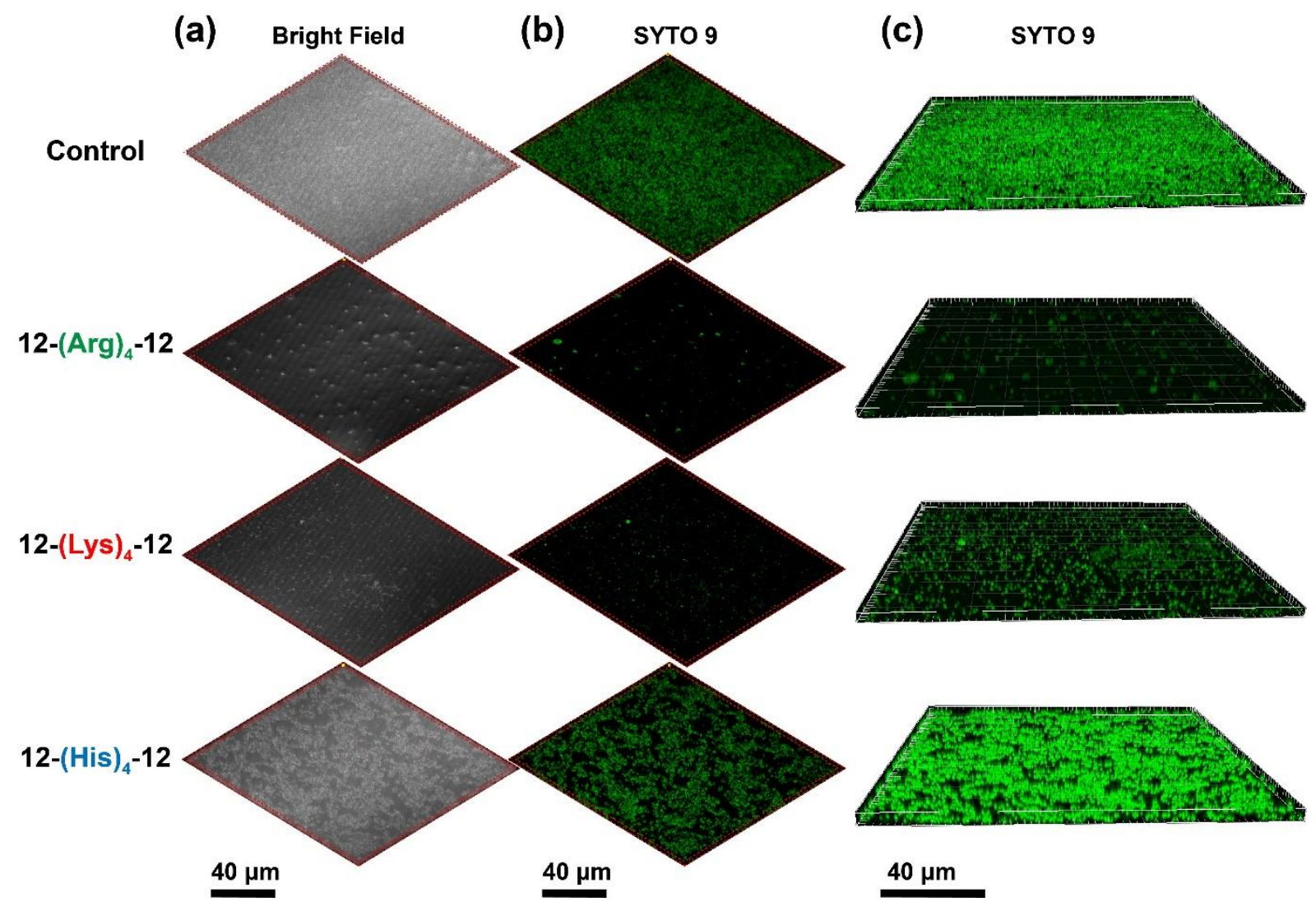

Figure S5. CLSM images of peptide amphiphiles-treated S. aureus biofilms. (a) Bright field, (b) SYTO 9 channel and (c) 3D images of SYTO 9 channel. Biofilms were grown for $24 \mathrm{~h}$ and then treated with PBS solution (control), or $50 \mu \mathrm{M}$ 12-(Arg)4-12, $100 \mu \mathrm{M}$ 12-(Lys)4-12, and $100 \mu \mathrm{M} 12$-(His) ${ }_{4}-12$ for $2 \mathrm{~h}$. Biofilms were stained with BacLight Live/Dead stain. 

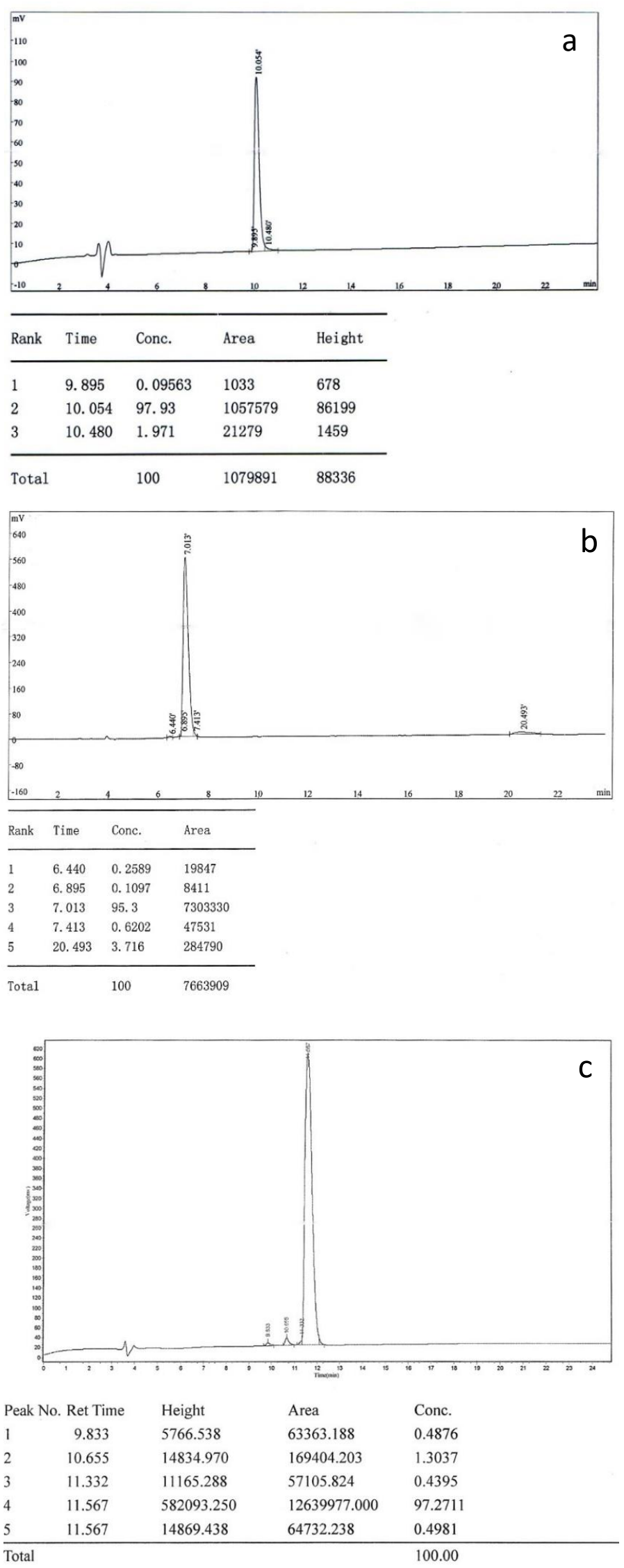

Figure S6. High-performance liquid chromatography of (a) 12-(Arg) $4-12$, (b) 12-(Lys) $4-12$ and (c) $12-(\mathrm{His})_{4}-12$. 
a
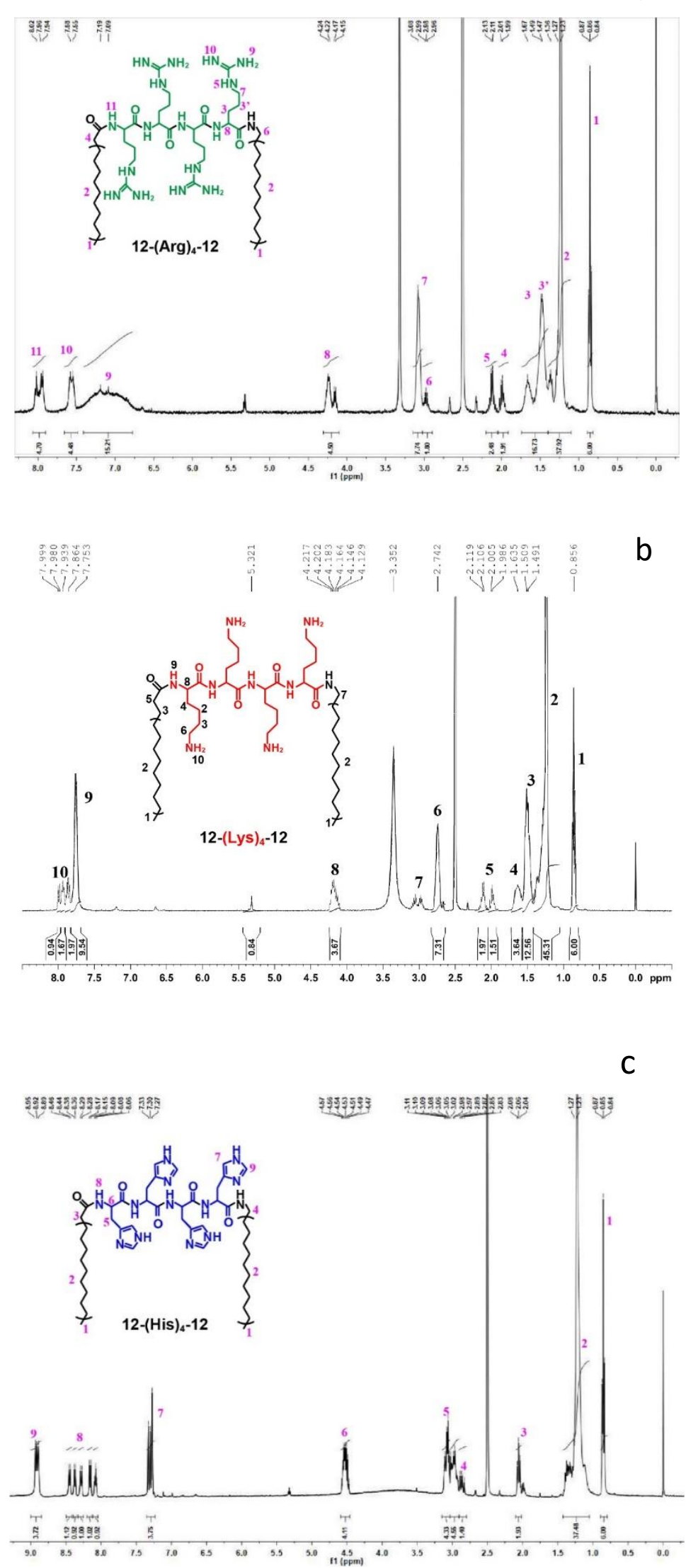

Figure S7. ${ }^{1} \mathrm{H}$ NMR spectra of (a) 12-(Arg) $)_{4}-12$, (b) 12-(Lys) $4-12$ and (c) 12-(His) $4-12$. 

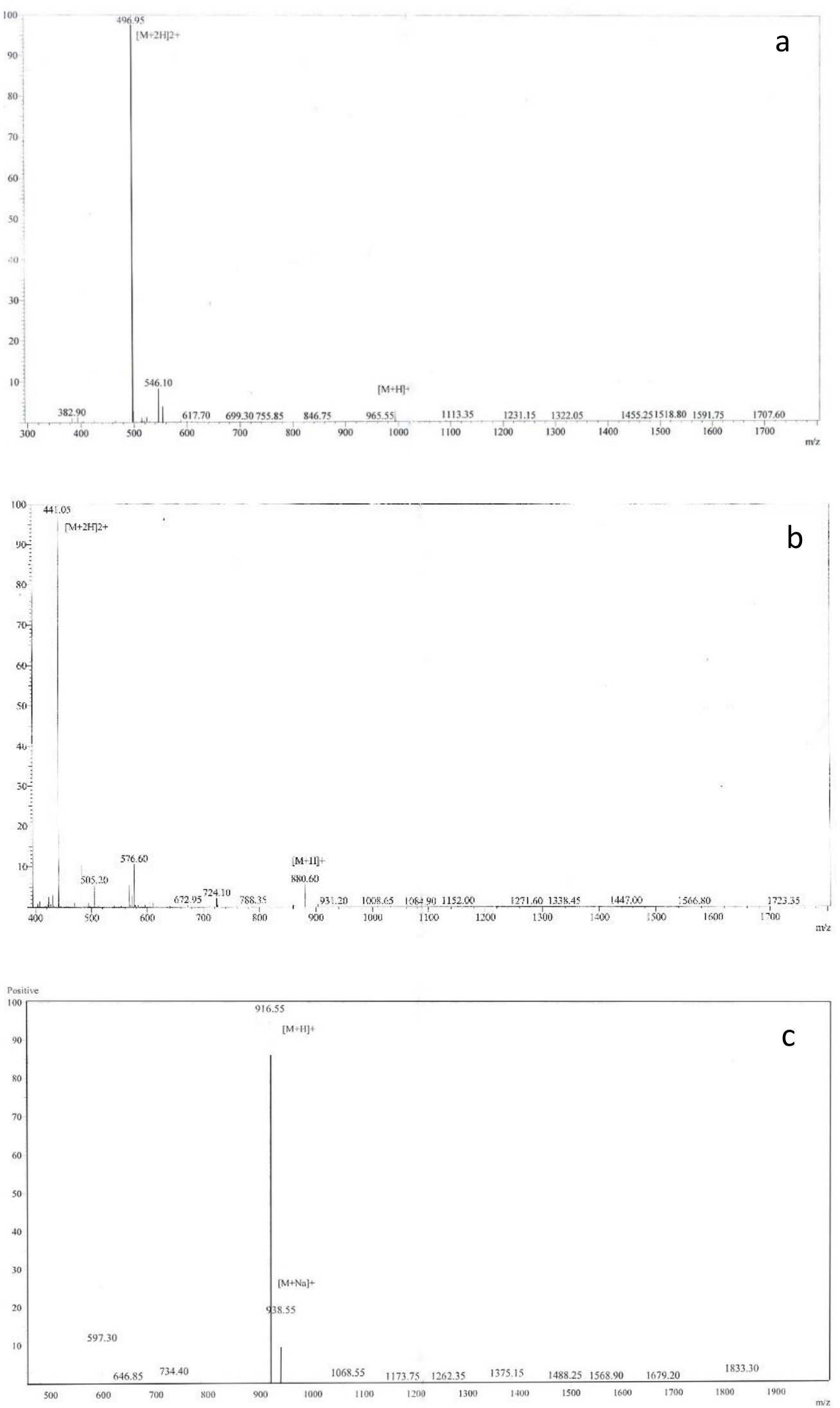

Figure S8. ESI mass spectra of (a) 12-(Arg) $4-12$, (b) 12-(Lys) $)_{4}-12$ and (c) 12-(His) $)_{4}-12$. 


\section{ITC Analysis Process for Single Set of Identical Sites.}

In the following equations,

$K_{\mathrm{b}}=$ binding constant;

$N=$ number of binding sites;

$\Theta=$ fraction of sites occupied by ligand (the titrant);

$M_{\mathrm{t}}$ and $[M]$ are bulk and free concentration of titrand placed in the sample cells in $V_{0}$;

$X_{\mathrm{t}}$ and $[X]$ are bulk and free concentration of ligand;

$V_{0}=$ active cell volume;

$\Delta V_{\mathrm{i}}=$ injection volume;

$Q=$ the heat content;

$Q_{\mathrm{i}}=$ heat content from the completion of the $\mathrm{i}^{\text {th }}$ injection .

The interaction process may be described in a simplified fashion using equation

$$
X+M \leftrightarrow X M \quad[1]
$$

In the process, the binding constant and the concentration of ligand can be expressed as follows:

$$
\begin{aligned}
K_{b} & =\frac{\Theta}{(1-\Theta[X])} \\
X_{t} & =[X]+N \Theta M_{t}
\end{aligned}
$$

Combining equations [2] and [3] above gives:

$$
\Theta^{2}-\Theta\left[1+\frac{X_{t}}{N M_{t}}+\frac{1}{N K M_{t}}\right]+\frac{X_{t}}{N M_{t}}=0
$$

The total heat content $Q$ of the solution contained in $V_{0}$ at fractional saturation $\Theta$ is:

$$
Q=N \Theta M_{t} \Delta H V_{0}
$$

Solving the quadratic equation [4] for $\Theta$ and then substituting this into equation [5] gives:

$$
Q=\frac{N M_{\mathrm{t}} \Delta H V_{0}}{2}\left[1+\frac{X_{\mathrm{t}}}{N M_{\mathrm{t}}}+\frac{1}{N K M_{\mathrm{t}}}-\sqrt{\left(1+\frac{X_{\mathrm{t}}}{N M_{\mathrm{t}}}+\frac{1}{N K M_{\mathrm{t}}}\right)^{2}-\frac{4 X_{\mathrm{t}}}{N M_{\mathrm{t}}}}\right]
$$

The heat $\Delta Q(i)$ released from the $i^{\text {th }}$ injection can be expressed as:

$$
\Delta Q(i)=Q(i)+\frac{d V_{\mathrm{t}}}{V_{0}}\left[\frac{Q(i)+Q(i-1)}{2}\right]-Q(i-1)
$$

which may be used in the Marquardt algorithm to obtain best values for the fitting parameters $N, K_{b}$ and $\Delta H$. More information about the model analysis can be found in ITC Data Analysis in Origin ${ }^{\circledR}$ Tutorial Guide Version 7.0. 